\title{
Pacific
}

Journal of

Mathematics

\section{ROOTS OF TOEPLITZ OPERATORS ON THE BERGMAN SPACE}

ISSAM LOUHICHI AND N. V. RAO 


\title{
ROOTS OF TOEPLITZ OPERATORS ON THE BERGMAN SPACE
}

\author{
ISSAM LOUHICHI AND N. V. RAO
}

\begin{abstract}
A major goal in the theory of Toeplitz operators on the Bergman space over the unit disk $\mathbb{D}$ in the complex plane $\mathbb{C}$ is to competely describe the commutant of a given Toeplitz operator, that is, the set of all Toeplitz operators that commute with it. In [2007], the first author characterized the commutant of a Toeplitz operator $T$ that has a quasihomogeneous symbol $\phi(r) e^{i p \theta}$ with $p>0$, in case it has a Toeplitz $p$-th $\operatorname{root} S$ with symbol $\psi(r) e^{i \theta}$ : The commutant of $T$ is the closure of the linear space generated by powers $S^{n}$ that are Toeplitz. But the existence of a $p$-th root was known until now only when $\phi(r)=r^{m}$ with $m \geq 0$. Here we will show the existence of $p$-th roots for a much larger class of symbols, for example, those symbols for which
\end{abstract}

$$
\phi(r)=\sum_{i=1}^{k} r^{a_{i}}(\ln r)^{b_{i}}, \quad \text { where } 0 \leq a_{i}, b_{i} \text { for all } 1 \leq i \leq k .
$$

\section{Introduction}

Let $\mathbb{D}$ be the unit disk in the complex plane $\mathbb{C}$, and let $d A=r d r d \theta / \pi$ be the Lebesgue area measure normalized so that $\mathbb{D}$ has unit measure. Let $L_{a}^{2}$ be the Bergman space, the Hilbert space of functions that are analytic on $\mathbb{D}$ and square integrable with respect to $d A$. We denote the inner product in $L^{2}(\mathbb{D}, d A)$ by $\langle\cdot, \cdot\rangle$. It is well known that $L_{a}^{2}$ is a closed subspace of the Hilbert space $L^{2}(\mathbb{D}, d A)$, and the set $\left\{\sqrt{n+1} z^{n} \mid n \geq 0\right\}$ of functions is an orthonormal basis. Let $P$ be the orthogonal projection from $L^{2}(\mathbb{D}, d A)$ onto $L_{a}^{2}$. For a bounded function $f$ on $\mathbb{D}$, the Toeplitz operator $T_{f}$ with symbol $f$ is defined by

$$
T_{f}(h)=P(f h) \quad \text { for } h \in L_{a}^{2} .
$$

A symbol $f$ is said to be quasihomogeneous of order $p$ an integer if it can be written as $f\left(r e^{i \theta}\right)=e^{i p \theta} \phi(r)$, where $\phi$ is a radial function on $\mathbb{D}$. In this case, the associated Toeplitz operator $T_{f}$ is also called quasihomogeneous Toeplitz of order $p$. Quasihomogeneous Toeplitz operators were first introduced in [Louhichi

MSC2000: primary 47B35; secondary 47L80.

Keywords: Toeplitz operators, Bergman space, Mellin transform, Gamma funtion, Beta function. 
and Zakariasy 2005] while generalizing the results of [Čučković and Rao 1998]. We assume $p>0$ from now on.

For a given a quasihomogeneous operator $T$ of degree $p$, we seek a quasihomogeneous operator $S$ of degree 1 such that $S^{p}=T$. Louhichi [2007] proved that if any such root exists, it is unique up to a multiplicative constant. Using the results in [Čučković and Rao 1998], Louchichi also proved the existence of $p$-th roots for the case $\phi(r)=r^{m}$ for any arbitrary $m \geq 0$ and $p>0$. Here we plan to deal with more general $\phi(r)$.

\section{The Mellin transform and two lemmas}

For any two functions $f(r)$ and $g(r)$ defined on $I=[0,1]$, we define the Mellin convolution by

$$
\left(f *_{M} g\right)(r)=\int_{r}^{1} f\left(\frac{r}{t}\right) g(t) \frac{d t}{t} .
$$

Often we are interested in knowing when the Mellin convolution is a bounded function in the interval $I$. We say a function $f$ is of type $(a, b)$ with $a \geq 0$ and $b>0$ if

$$
|f(r)| \leq C r^{a}(1-r)^{b-1} \quad \text { on } I,
$$

where $C$ is a constant depending on $f$. Also we express the same thing as

$$
f(r) \ll r^{a}(1-r)^{b-1},
$$

omitting the constants and the absolute value signs.

Lemma A. Suppose $f(r)$ is of type $(a, b)$ and $g(r)$ is of type $(c, d)$. Then

$$
\begin{cases}\left(f *_{M} g\right) \quad \text { is of type } \quad(\min \{a, c\}, b+d) & \text { if } a \neq c, \text { and } \\ \left(f *_{M} g\right)(r) \ll r^{\min \{a, c\}}(1-r)^{b+d-1} \ln (e / r) & \text { if } a=c .\end{cases}
$$

This can be generalized to any finite product as follows: Suppose for $1 \leq i \leq n$, $f_{i}(r)$ is of type $\left(a_{i}, b_{i}\right)$. Then their Mellin convolution product $h(r)$ satisfies

$$
h(r) \ll r^{\alpha}(1-r)^{\beta-1}\left(\ln \left(\frac{e}{r}\right)\right)^{n-1}
$$

where $\alpha=\min \left\{a_{i}\right\}$ and $\beta=\sum b_{i}$. Further, if we know that the number of $a_{i}$ that are equal to $\min \left\{a_{i}\right\}$ is (say) $l$, the estimate (1) can be improved to

$$
h(r) \ll r^{\alpha}(1-r)^{\beta-1}\left(\ln \left(\frac{e}{r}\right)\right)^{l-1} .
$$

Thus the log term will disappear if $l=1$.

Remark 2.1. Most of the time our aim is to prove $h$ is bounded; the presence of $\log$ does not interfere with that aim since $\alpha>0$, which bounds $h(r)$ near zero, 
and if we assume further that $\beta \geq 1$, it would be bounded near 1 also. But log cannot be avoided. Take for example $f_{i}(r)=r$ for every $i$ and compute the Mellin convolution product. It turn out to be $r(\ln r)^{n-1} /(n-1)$ !, by a simple integration.

Lemma B. Let $f_{i}(r)=r^{a_{i}}(1-r)^{b_{i}-1}$, where $a_{i}$ and $b_{i}$ are positive for $1 \leq i \leq n$. Let $\alpha$ and $\beta$ be as defined in Lemma A. Let $h$ be the Mellin covolution product of the $f_{i}$. For any integer $k \geq 0$, the $k$-th derivative of $h$ satisfies

$$
h^{(k)}(r) \ll r^{\alpha-k}(1-r)^{\beta-k-1}\left(\ln \left(\frac{e}{r}\right)\right)^{n-1} .
$$

Here the implied constant depends on $k$ and $h$.

\section{Applications of Lemmas A and B}

The Mellin transform $\hat{\phi}$ of a radial function $\phi$ in $L^{1}([0,1], r d r)$ is defined by

$$
\hat{\phi}(z)=\int_{0}^{1} \phi(r) r^{z-1} d r=\mathcal{M}(\phi)(z) .
$$

It is well known that for these functions the Mellin transform is well-defined on the right half-plane $\{z: \operatorname{Re} z \geq 2\}$ and analytic on $\{z: \operatorname{Re} z>2\}$. The Mellin transform $\hat{\phi}$ is uniquely determined by its values on any arithmetic sequence of integers. In fact we have the following classical theorem [Remmert 1998, page 102].

Theorem 3.1. Suppose $f$ is a bounded analytic function on $\{z: \operatorname{Re} z>0\}$ that vanishes at the pairwise distinct points $z_{1}, z_{2}, \ldots$, where

(1) $\inf \left\{\left|z_{n}\right|\right\}>0$ and

(2) $\sum_{n \geq 1} \operatorname{Re}\left(1 / z_{n}\right)=\infty$.

Then $f$ vanishes identically on $\{z: \operatorname{Re} z>0\}$.

Remark 3.2. One can apply this theorem to prove that if $\phi \in L^{1}([0,1], r d r)$ and if there exist $n_{0}, p \in \mathbb{N}$ such that

$$
\hat{\phi}\left(p k+n_{0}\right)=0 \quad \text { for all } k \in \mathbb{N},
$$

then $\hat{\phi}(z)=0$ for all $z \in\{z: \operatorname{Re} z>2\}$ and so $\phi=0$.

It is easy to see that the Mellin transform converts the Mellin convolution product into a pointwise product, that is,

$$
\left(\widehat{\phi *_{M} \psi}\right)(r)=\hat{\phi}(r) \hat{\psi}(r) .
$$

A direct calculation shows that a quasihomogeneous Toeplitz operator acts on the elements of the orthogonal basis of $L_{a}^{2}$ as a shift operator with a holomorphic 
weight. In fact, for $p \geq 0$ and for all $k \geq 0$, we have

$$
\begin{aligned}
T_{e^{i p \theta} \phi}\left(z^{k}\right)=P\left(e^{i p \theta} \phi z^{k}\right) & =\sum_{n \geq 0}(n+1)\left\langle e^{i p \theta} \phi z^{k}, z^{n}\right\rangle z^{n} \\
& =\sum_{n \geq 0}(n+1) \int_{0}^{1} \int_{0}^{2 \pi} \phi(r) r^{k+n+1} e^{i(k+p-n) \theta} \frac{d \theta}{\pi} d r z^{n} \\
& =2(k+p+1) \hat{\phi}(2 k+p+2) z^{k+p} .
\end{aligned}
$$

Now we are ready to start with a relatively easy example.

3.1. Assuming $\phi(r)=r+r^{2}$, find the p-th roots of $T_{e^{i p \theta} \phi}$. If there exists a bounded radial function $\psi$ such that $\left(T_{e^{i \theta} \psi}\right)^{p}=T_{e^{i p \theta} \phi}$, then

$$
\left(T_{e^{i \theta} \psi}\right)^{p}\left(z^{k}\right)=T_{e^{i p \theta} \phi}\left(z^{k}\right) \quad \text { for all } k \geq 0 .
$$

Since

$$
\left(T_{e^{i \theta} \psi}\right)^{p}\left(z^{k}\right)=\left(\prod_{j=0}^{p-1}(2 k+2 j+4) \psi(2 k+2 j+3)\right) z^{k+p},
$$

we obtain for all integers $k \geq 0$

$$
(2 k+2 p+2) \hat{\phi}(2 k+p+2)=\left(\prod_{j=0}^{p-1}(2 k+2 j+4) \hat{\psi}(2 k+2 j+3)\right),
$$

which is equivalent to

$$
\frac{\hat{\phi}(2 k+p+2)}{\prod_{j=0}^{p-2}(2 k+2 j+4)}=\prod_{j=0}^{p-1} \hat{\psi}(2 k+2 j+3) .
$$

Note that $p$ is a positive integer and that our discussion is trivial for $p=1$. So $p \geq 2$. By setting $z=2 k+3$, we notice that the function

$$
f(z)=\frac{\hat{\phi}(z+p-1)}{\prod_{j=0}^{p-2}(z+2 j+1)}-\prod_{j=0}^{p-1} \hat{\psi}(z+2 j)
$$

is holomorphic and bounded in the right half-plane and vanishes for $z=2 k+3$, for $k$ any nonnegative integer. Now by Theorem 3.1, we get $f(z) \equiv 0$. Therefore

$$
(z+2 p-1) \hat{\phi}(z+p-1)=\left(\prod_{j=0}^{p-1}(z+2 j+1) \hat{\psi}(z+2 j)\right) .
$$


If we divide the (2) by the equation obtained by replacing $z$ by $z+2$ in (2), we obtain after cancelation that in the right half-plane

$$
\frac{\hat{\psi}(z+2 p)}{\hat{\psi}(z)}=\frac{(z+1) \hat{\phi}(z+p+1)}{(z+2 p-1) \hat{\phi}(z+p-1)} \quad \text { for } \operatorname{Re} z>0 .
$$

Since

$$
\hat{\phi}(z)=\frac{1}{z+1}+\frac{1}{z+2}=\frac{2 z+3}{(z+1)(z+2)},
$$

it follows that, for $\operatorname{Re} z>0$,

$$
\frac{\hat{\psi}(z+2 p)}{\hat{\psi}(z)}=\frac{(z+1)}{(z+2 p-1)} \frac{(2 z+2 p+5)}{(z+p+2)(z+p+3)} \frac{(z+p)(z+p+1)}{(2 z+2 p+1)} .
$$

Letting $\lambda(\zeta)=\hat{\psi}(2 p \zeta)$, this equation becomes, for $\operatorname{Re} \zeta>0$,

$$
\frac{\lambda(\zeta+1)}{\lambda(\zeta)}=\frac{(2 p \zeta+1)(4 p \zeta+2 p+5)(2 p \zeta+p)(2 p \zeta+p+1)}{(2 p \zeta+2 p-1)(2 p \zeta+p+2)(2 p \zeta+p+3)(4 p \zeta+2 p+1)} .
$$

Using the well-known identity $\Gamma(z+1)=z \Gamma(z)$, where $\Gamma$ is the Gamma function, we can write

$$
\frac{\lambda(\zeta+1)}{\lambda(\zeta)}=\frac{F(\zeta+1)}{F(\zeta)} \text { for } \operatorname{Re} \zeta>0
$$

where

$$
F(\zeta)=\frac{\Gamma\left(\zeta+a_{1}\right) \Gamma\left(\zeta+a_{2}\right) \Gamma\left(\zeta+a_{3}\right) \Gamma\left(\zeta+a_{4}\right)}{\Gamma\left(\zeta+a_{1}^{\prime}\right) \Gamma\left(\zeta+a_{2}^{\prime}\right) \Gamma\left(\zeta+a_{3}^{\prime}\right) \Gamma\left(\zeta+a_{4}^{\prime}\right)}
$$

and the $a_{i}$ are in increasing order

$$
\frac{2}{4 p}, \quad \frac{2 p}{4 p}, \quad \frac{2 p+2}{4 p}, \frac{2 p+5}{4 p}
$$

respectively and the $a_{i}^{\prime}$ are in almost increasing order

$$
\frac{2 p+1}{4 p}, \frac{2 p+4}{4 p}, \frac{4 p-2}{4 p}, \frac{2 p+6}{4 p}
$$

respectively for $i=1, \ldots, 4$. We shall show in a moment that $F(\zeta)$ is a bounded holomorphic function in the right half-plane. Granting that, Equation (4) combined with [Louhichi 2007, Lemma 6, page 1468] implies exists a constant $C$ such that

$$
\lambda(\zeta)=C F(\zeta) \text { for } \operatorname{Re} \zeta>0 .
$$

A basic observation is that the quotient of two Gamma functions

$$
\frac{\Gamma\left(\zeta+a_{i}\right)}{\Gamma\left(\zeta+a_{i}^{\prime}\right)}, \quad \text { where } 0<a_{i}<a_{i}^{\prime},
$$


is a constant times the Beta function

$$
B\left(\zeta+a_{i}, a_{i}^{\prime}-a_{i}\right)=\int_{0}^{1} x^{\zeta+a_{i}-1}(1-x)^{a_{i}^{\prime}-a_{i}-1} d x .
$$

According to our definition of the Mellin transform, $B\left(\zeta+a_{i}, a_{i}^{\prime}-a_{i}\right)$ is the Mellin transform of $x^{a_{i}}(1-x)^{a_{i}^{\prime}-a_{i}-1}$, which is of type $\left(a_{i}, a_{i}^{\prime}-a_{i}\right)$. Since $a_{i}<a_{i}^{\prime}$ for $i=1, \ldots, 4$ (in fact, $a_{3}^{\prime} \geq a_{3}$ if and only if $2 p \geq 4$, which is always true), each of the Beta functions is a bounded holomorphic function in the right half-plane and $F(\zeta)$, which is a constant times the product of these four Beta functions, is a bounded holomorphic function in the right half-plane. Equation (5) implies that

$$
\lambda(\zeta)=C \sum_{i=1}^{4} B\left(\zeta+a_{i}, a_{i}^{\prime}-a_{i}\right),
$$

where $C$ is a constant. Since the product of Mellin transforms equals the Mellin transform of the Mellin convolution product, we have

$$
\lambda(\zeta)=C h(\zeta)
$$

where $h$ is the convolution product of four functions of type $\left(a_{i}, a_{i}^{\prime}-a_{i}\right)$ for $i=$ $1, \ldots, 4$. Now Lemma A tells us that

$$
h(r) \ll r^{\min \left\{a_{i}\right\}}(1-r)^{\sum_{i}\left(a_{i}^{\prime}-a_{i}\right)-1} \ln (e / r) .
$$

Because $\sum_{i} a_{i}^{\prime}-a_{i}=1$, we have

$$
h(r) \ll r^{\min \left\{a_{i}\right\}} \ln (e / r),
$$

and hence $h$ is a bounded function. Therefore the function $\psi$, if it exists, satisfies the equation

$$
\hat{\psi}(2 p \zeta)=C \hat{h}(\zeta)
$$

for some constant $C$, which is equivalent to

$$
\int_{0}^{1} \psi(r) r^{2 p \zeta-1} d r=C \int_{0}^{1} h(t) t^{\zeta-1} d t
$$

Now, by a change of variables $t=r^{2 p}$, we obtain

$$
\int_{0}^{1} \psi(r) r^{2 p \zeta-1} d r=\int_{0}^{1} h\left(r^{2 p}\right) r^{2 p \zeta-1} 2 p d r .
$$

Thus $\psi(r)=2 p h\left(r^{2 p}\right)$, and so $\psi$ is bounded. Hence the operator $T_{e^{i \theta} \psi}$ is a genuine Toeplitz operator and a $p$-th root of $T_{e^{i p \theta} \phi}$. 
3.2. $p$-th roots of $T_{e^{i p \theta} \phi}$, where $\hat{\phi}(z)$ is a proper rational fraction. Such functions are plenty. For example, take $\Phi(r)=r^{a} \ln (r)^{b}$, where $a>0$ and $b$ is a nonnegative integer. By integration by parts we see that $\hat{\Phi}(z)=(-1)^{b} b ! /(a+z)^{b+1}$.

Assume we are given a radial function $\phi(r)$ such that $\hat{\phi}(r)$ is a proper rational function. Recall that if there is a radial function $\psi$ such that $\left(T_{e^{i \theta} \psi}\right)^{p}=T_{e^{i p \theta} \phi}$, then we have Equation (3), which is

$$
\hat{\psi}(z+2 p)=\hat{\psi}(z) \frac{(z+1) \hat{\phi}(z+p+1)}{(z+2 p-1) \hat{\phi}(z+p-1)} \quad \text { for } \operatorname{Re} z>0 .
$$

Here we are assuming $\hat{\phi}(z)=P(z) / Q(z)$, where

$$
P(z)=\prod_{j=1}^{m}\left(z+a_{j}\right) \quad \text { and } \quad Q(z)=\prod_{k=1}^{n}\left(z+b_{k}\right)
$$

with $1 \leq m<n$. So,

$$
\begin{aligned}
\hat{\psi}(z+2 p) & =\hat{\psi}(z) \frac{(z+1)}{(z+2 p-1)} \frac{P(z+p+1) Q(z+p-1)}{P(z+p-1) Q(z+p+1)} \\
& =\frac{(z+1)}{(z+2 p-1)} \prod_{j=1}^{m} \frac{z+a_{j}+p+1}{z+a_{j}+p-1} \prod_{k=1}^{n} \frac{z+b_{k}+p-1}{z+b_{k}+p+1}
\end{aligned}
$$

Let $\lambda(\zeta)=\hat{\psi}(2 p \zeta)$. Then the equality above becomes

$$
\begin{aligned}
\frac{\lambda(\zeta+1)}{\lambda(\zeta)} & =\frac{(2 p \zeta+1)}{(2 p \zeta+2 p-1)} \prod_{j=1}^{m} \frac{2 p \zeta+a_{j}+p+1}{2 p \zeta+a_{j}+p-1} \prod_{k=1}^{n} \frac{2 p \zeta+b_{k}+p-1}{2 p \zeta+b_{k}+p+1} \\
& =\frac{F(\zeta+1) G(\zeta)}{F(\zeta) G(\zeta+1)}
\end{aligned}
$$

where

$$
\begin{aligned}
F(\zeta) & =\frac{\Gamma\left(\zeta+A_{0}\right)}{\Gamma\left(\zeta+A_{0}^{\prime}\right)} \prod_{k=1}^{n} \frac{\Gamma\left(\zeta+B_{k}\right)}{\Gamma\left(\zeta+B_{k}^{\prime}\right)} \quad \text { and } \quad G(\zeta)=\prod_{j=1}^{m} \frac{\Gamma\left(\zeta+A_{j}^{\prime}\right)}{\Gamma\left(\zeta+A_{j}\right)}, \\
A_{0} & =\frac{1}{2 p}, \quad A_{0}^{\prime}=\frac{2 p-1}{2 p}, \\
A_{j} & =\frac{a_{j}+p+1}{2 p}, \quad A_{j}^{\prime}=\frac{a_{j}+p-1}{2 p}, \\
B_{k} & =\frac{b_{k}+p-1}{2 p}, \quad B_{k}^{\prime}=\frac{b_{k}+p+1}{2 p} \quad \text { for } 1 \leq j \leq m \text { and } 1 \leq k \leq n .
\end{aligned}
$$


Note that any quotient of two Gamma functions, say,

$$
\frac{\Gamma(\zeta+\alpha)}{\Gamma(\zeta+\gamma)}=\beta(\zeta+\alpha, \gamma-\alpha) \Gamma(\gamma-\alpha)
$$

is a bounded holomorphic function in the right half-plane if $\alpha$ and $\gamma-\alpha$ are positive. Hence both $F(\zeta)$ and $G(\zeta)$ are bounded holomorphic functions in the right halfplane if we assume all $A_{j}, A_{j}^{\prime}, B_{k}, B_{k}^{\prime}$ are positive. We will assume that.

Therefore, by [Louhichi 2007, Lemma 6 , page 1468], $\lambda$ is a constant times the quotient of $m+n+1$ Gamma functions in the numerator and about the same in the denominator, as follows:

$$
\lambda(\zeta)=C \frac{\Gamma\left(\zeta+A_{0}\right)}{\Gamma\left(\zeta+A_{0}^{\prime}\right)} \prod_{j=1}^{m} \frac{\Gamma\left(\zeta+A_{j}\right)}{\Gamma\left(\zeta+A_{j}^{\prime}\right)} \prod_{k=1}^{n} \frac{\Gamma\left(\zeta+B_{k}\right)}{\Gamma\left(\zeta+B_{k}^{\prime}\right)} .
$$

Based on the argument of the previous subsection, we would like to write each quotient of two Gamma functions as a constant times a Beta function. In order to do that, we must assume that all $A_{j}$ and $B_{k}$ are positive for every $0 \leq j \leq m$ and $1 \leq k \leq n$. Moreover, we observe that

$$
A_{0}^{\prime}-A_{0}=\frac{p-1}{p}, \quad A_{j}^{\prime}-A_{j}=-\frac{1}{p}, \quad B_{k}^{\prime}-B_{k}=\frac{1}{p} .
$$

So each quotient of two Gamma functions in Equation (6) can be written as a constant times a Beta function except those involving $A_{j}$ for $1 \leq j \leq m$. We fix this matter by noting that $\Gamma\left(\zeta+A_{j}^{\prime}+1\right)=\left(\zeta+A_{j}^{\prime}\right) \Gamma\left(\zeta+A_{j}^{\prime}\right)$, and so here $A_{j}^{\prime}+1-A_{j}=(p-1) / p$. Hence, Equation (6) becomes

$$
\frac{\lambda(\zeta)}{\prod_{j=1}^{m}\left(\zeta+A_{j}^{\prime}\right)}=C \frac{\Gamma\left(\zeta+A_{0}\right)}{\Gamma\left(\zeta+A_{0}^{\prime}\right)} \prod_{j=1}^{m} \frac{\Gamma\left(\zeta+A_{j}\right)}{\Gamma\left(\zeta+A_{j}^{\prime}+1\right)} \prod_{j=1}^{n} \frac{\Gamma\left(\zeta+B_{j}\right)}{\Gamma\left(\zeta+B_{j}^{\prime}\right)}
$$

As in the previous subsection, this quotient of $m+n+1$ Gamma functions on the numerator and the same in the denominator, respectively would be the Mellin transform of the convolution product of $m+n+1$ functions of type $\left(a_{i}, b_{i}\right)$. Let us call it $h$. By Lemma A, we have

$$
h(r) \ll r^{A}(1-r)^{B-1}\left(\ln \left(\frac{e}{r}\right)\right)^{m+n},
$$

where $A=\min \left\{A_{j}\right\}$, which is definitely positive, and $B$ is given by

$$
A_{0}^{\prime}-A_{0}+\sum_{j=1}^{m} A_{j}^{\prime}+1-A_{j}+\sum_{k=1}^{n} B_{k}^{\prime}-B_{k}=(m+1) \frac{p-1}{p}+\frac{n}{p}=m+1+\frac{n-m-1}{p} \text {. }
$$


Therefore we obtain

$$
h(r) \ll r^{A}(1-r)^{m+(n-m-1) / p}\left(\ln \left(\frac{e}{r}\right)\right)^{m+n}=r^{A}(1-r)^{m+v}\left(\ln \left(\frac{e}{r}\right)\right)^{m+n},
$$

where $v=(n-m-1) / p$ is a nonnegative number. Using Lemma $\mathrm{B}$, we see that $h$ has all derivatives of order not exceeding $m$ and they satisfy the inequality

$$
r^{j} h^{(j)}(r) \ll r^{A}(1-r)^{m-j+v}\left(\ln \left(\frac{e}{r}\right)\right)^{m+n} .
$$

Further the function $\psi$, were it to exist, would satisfy the equation

$$
\hat{\psi}(2 p \zeta)=C\left(\prod_{j=1}^{m}\left(\zeta+A_{j}^{\prime}\right)\right) \hat{h}(\zeta) .
$$

Now it is easy to check by integration by parts the identity

$$
\zeta \hat{h}(\zeta)=-\mu\left(r \frac{d h}{d r}\right)(\zeta)
$$

provided $h$ vanishes at 1 and $r h^{\prime}$ is bounded in $(0,1)$. Thus in the current case, letting $h^{\prime}=D h$, where $D=d / d r$, we can see

$$
\left(\zeta+A_{j}^{\prime}\right) \hat{h}(\zeta)=M\left(\left(A_{j}^{\prime}-r D\right) h\right)(\zeta)
$$

and

$$
\left(\prod_{j=1}^{m}\left(\zeta+A_{j}^{\prime}\right)\right) \hat{h}(\zeta)=M\left(\prod_{j=1}^{m}\left(A_{j}^{\prime}-r D\right) h\right)(\zeta) .
$$

Let us set

$$
H(r)=\left(\prod_{j=1}^{m}\left(A_{j}^{\prime}-r D\right) h\right)(r),
$$

which allows us to rewrite Equation (7) as

$$
\int_{0}^{1} \psi(r) r^{2 p \zeta-1} d r=C \int_{0}^{1} H(t) t^{\zeta-1} d t
$$

Now, by a change of variables $t=r^{2 p}$, we obtain

$$
\int_{0}^{1} \psi(r) r^{2 p \zeta-1} d r=C \int_{0}^{1} H\left(r^{2 p}\right) r^{2 p \zeta-1} 2 p d r .
$$

Thus $\psi(r)=2 p C H\left(r^{2 p}\right)$, and hence is bounded and the operator $T_{e^{i \theta} \psi}$ is a genuine Toeplitz operator and a $p$-th root of $T_{e^{i p \theta} \phi}$. 


\section{Proof of Lemma A for two functions}

We start by proving Lemma A for functions $f$ and $g$ of type $(a, b)$ and $(c, d)$ respectively, where $a, b, c$ and $d$ are all positive. A similar thing was discussed in [Cucucković and Rao 1998, pages 210-212] but with less generality since the goal was different.

Let $h(r)=\left(f *_{M} g\right)(r)$. By definition of the Mellin convolution, it is easy to see that

$$
h(r) \ll \int_{r}^{1}\left(\frac{r}{t}\right)^{a}\left(1-\frac{r}{t}\right)^{b-1} t^{c}(1-t)^{d-1} \frac{d t}{t},
$$

which after changing variables as $\frac{t-r}{1-r}=u$ and using the consequent identities

$$
t=r+u-r u, \quad t-r=u(1-r), \quad 1-t=(1-u)(1-r), \quad d t=(1-r) d u
$$

while keeping $r$ fixed, leads to

$$
\begin{aligned}
h(r) & \ll \int_{r}^{1}\left(\frac{r}{t}\right)^{a}\left(1-\frac{r}{t}\right)^{b-1} t^{c}(1-t)^{d-1} \frac{d t}{t} \\
& =\int_{r}^{1}\left(\frac{r}{t}\right)^{a}\left(\frac{t-r}{t}\right)^{b-1} t^{c}(1-t)^{d-1} \frac{d t}{t} \\
& =\int_{0}^{1} r^{a} t^{-a} u^{b-1}(1-r)^{b-1} t^{-b+1} t^{c}(1-u)^{d-1}(1-r)^{d-1}(1-r) \frac{d u}{t} \\
& =r^{a}(1-r)^{b+d-1} \int_{0}^{1} t^{c-a-b} u^{b-1}(1-u)^{d-1} d u .
\end{aligned}
$$

We have the following cases.

- $c-a-b \geq 0$. Since $0 \leq t \leq 1$, we have

$$
h(r) \ll r^{a}(1-r)^{b+d-1},
$$

and hence $h$ is of type $(a, b+d)$.

- $c-a-b<0$. Assuming $c-a>0$ and noting that $t \geq u$, we obtain

$$
\begin{aligned}
h(r) & \ll r^{a}(1-r)^{b+d-1} \int_{0}^{1} u^{c-a-b} u^{b-1}(1-u)^{d-1} d u \\
& \leq r^{a}(1-r)^{b+d-1} \int_{0}^{1} u^{c-a-1}(1-u)^{d-1} d u \\
& =r^{a}(1-r)^{b+d-1} B(c-a, d),
\end{aligned}
$$

and therefore $h$ is of type $(a, b+d)$. 
Now in case $c=a$, consider any number $\epsilon$ in $0<\epsilon \leq b$. Noticing that $t \geq r$ and $u>0$, we have

$$
\begin{aligned}
h(r) & \ll r^{a}(1-r)^{b+d-1} \int_{0}^{1} t^{-b} u^{b-1}(1-u)^{d-1} d u \\
& \ll r^{a}(1-r)^{b+d-1} \int_{0}^{1} t^{-\epsilon} t^{\epsilon-b} u^{b-1}(1-u)^{d-1} d u \\
& \leq r^{a}(1-r)^{b+d-1} \int_{0}^{1} r^{-\epsilon} u^{\epsilon-b} u^{b-1}(1-u)^{d-1} d u \\
& \leq r^{a}(1-r)^{b+d-1} r^{-\epsilon} \int_{0}^{1} u^{\epsilon-1}(1-u)^{d-1} d u \\
& \leq r^{a}(1-r)^{b+d-1} B(\epsilon, d) r^{-\epsilon} .
\end{aligned}
$$

Now since $\epsilon B(\epsilon, d)=\Gamma(\epsilon+1) \Gamma(d) / \Gamma(\epsilon+d)$ is holomorphic as a function of $\epsilon$ in a neighborhood of the interval $(0, b)$, there exists a constant $C$ such that $\epsilon B(\epsilon, d) \leq C$ on that interval, and therefore

$$
h(r) \leq C r^{a}(1-r)^{b+d-1} r^{-\epsilon} \epsilon^{-1} \text { for every } 0<\epsilon \leq b .
$$

Here we emphasize the fact that $C$ does not depend on $r$ and $\epsilon$ as long as $0<r<1$ and $0<\epsilon \leq b$. For a fixed but arbitrary $r$, let $E(\epsilon)=r^{-\epsilon} \epsilon^{-1}$ and $m(r)=\min _{(0, b]} E(\epsilon)$. Then

$$
h(r) \leq C r^{a}(1-r)^{b+d-1} m(r) .
$$

Moreover the function $E$ decreases in the interval $(0,-1 / \ln r)$ and increases in the interval $(-1 / \ln r,+\infty)$. Further $-1 / \ln r \leq b$ if and only if $r \leq e^{-1 / b}$. Thus Equation (8) implies that

$$
\begin{array}{ll}
h(r) \ll r^{a}(1-r)^{b+d-1} m(r) \leq r^{a}(1-r)^{b+d-1} e \ln (1 / r) & \text { if } r \leq e^{-1 / b}, \\
h(r) \ll r^{a}(1-r)^{b+d-1} r^{-b} b^{-1} \leq r^{a}(1-r)^{b+d-1} e / b & \text { if } r>e^{-1 / b},
\end{array}
$$

Combining these two results, we obtain

$$
\begin{aligned}
h(r) & \ll r^{a}(1-r)^{b+d-1}(e \ln (1 / r)+e / b) \\
& \ll r^{a}(1-r)^{b+d-1} \ln (e / r) \text { for all } 0<r<1 .
\end{aligned}
$$

\section{Lemma A for the Mellin convolution product of more than two functions}

In this context we can assume that the function $f_{i}$, of type $\left(a_{i}, b_{i}\right)$, is given by

$$
f_{i}(x)=x^{a_{i}}(1-x)^{b_{i}-1} \quad \text { for } 1 \leq i \leq n .
$$


The Mellin convolution product of these $n$ functions is defined by a repeated integral

$$
\text { (9) } \begin{aligned}
h(r)= & \int_{r}^{1} \int_{r / x_{1}}^{1} \int_{r / x_{1} x_{2}}^{1} \cdots \int_{r / x_{1} x_{2} \cdots x_{n-2}}^{1} \\
& f_{1}\left(x_{1}\right) f_{2}\left(x_{2}\right) \cdots f_{n-1}\left(x_{n-1}\right) f_{n}\left(\frac{r}{x_{1} \cdots x_{n-1}}\right) \frac{d x_{n-1}}{x_{n-1}} \cdots \frac{d x_{3}}{x_{3}} \frac{d x_{2}}{x_{2}} \frac{d x_{1}}{x_{1}}
\end{aligned}
$$

As in the case of two functions where we changed variables as $u=(t-r) /(1-r)$, we change variables so that the new integral is over the unit cube $I^{n-1}$, where limits of integration do not depend on other variables. Let $y_{0}=1$ and inductively define $y_{i}=\prod_{j=1}^{i} x_{i}$ for $i \geq 1$. Now we change variables as

$$
x_{i}=\frac{r}{y_{i-1}}+\left(1-\frac{r}{y_{i-1}}\right) \xi_{i} \quad \text { for } i \geq 1,
$$

so that the limits for each $\xi_{i}$ are 0 and 1 . Further we note

$$
y_{i}-r=x_{i} y_{i-1}-r=\left(y_{i-1}-r\right) \xi_{i} \quad \text { for } i \geq 0 .
$$

Set $\eta_{0}=1$ and $\eta_{i}=\prod_{j=1}^{i} \xi_{i}$ for $i \geq 1$. It is easy to show, by induction on $i$, that

$$
y_{i}-r=(1-r) \eta_{i} \quad \text { for all } i \geq 1 .
$$

Further

$$
\left(1-x_{i}\right)=\left(1-\xi_{i}\right)\left(1-\frac{r}{y_{i-1}}\right)=\frac{\left(1-\xi_{i}\right)(1-r) \eta_{i-1}}{y_{i-1}} \quad \text { for all } i \geq 1 .
$$

Thus

$f_{i}\left(x_{i}\right)=x_{i}^{a_{i}}\left(1-x_{i}\right)^{b_{i}-1}=\left(\frac{y_{i}}{y_{i-1}}\right)^{a_{i}}\left(\frac{\left(1-\xi_{i}\right)(1-r) \eta_{i-1}}{y_{i-1}}\right)^{b_{i}-1} \quad$ for $1 \leq i \leq n-1$.

But for $i=n$, we have

$$
\begin{aligned}
f_{n}\left(x_{n}\right) & =\left(\frac{r}{y_{n-1}}\right)^{a_{n}}\left(1-\frac{r}{y_{n-1}}\right)^{b_{n}-1} \\
& =\left(\frac{r}{y_{n-1}}\right)^{a_{n}}\left(\frac{y_{n-1}-r}{y_{n-1}}\right)^{b_{n}-1}=\left(\frac{r}{y_{n-1}}\right)^{a_{n}}\left(\frac{(1-r) \eta_{n-1}}{y_{n-1}}\right)^{b_{n}-1} .
\end{aligned}
$$

Writing the product of functions in (9) in terms of $\xi_{i}, \eta_{i}, r$ and $y_{i}$ for $1 \leq i \leq n-1$ yields

$$
r^{a_{n}} \prod_{i=1}^{n-1} \eta_{i}^{b_{i+1}-1} \prod_{i=1}^{n-1} y_{i}^{a_{i}-a_{i+1}-b_{i+1}+1} \prod_{i=1}^{n-1}\left(1-\xi_{i}\right)^{b_{i}-1} \prod_{i=1}^{n}(1-r)^{b_{i}-1} .
$$


Using equalities of (10), we calculate the differential form

$$
\bigwedge_{i=1}^{n-1} \frac{d x_{i}}{x_{i}}=\bigwedge_{i=1}^{n-1} \frac{(1-r) \eta_{i-1} d \xi_{i}}{x_{i} y_{i-1}}=(1-r)^{n-1} \prod_{i=1}^{n-1} \frac{\eta_{i-1}}{y_{i}} \bigwedge_{i=1}^{n-1} d \xi_{i}
$$

From (9), (10), and (11) we derive

$$
\begin{aligned}
h(r)=r^{a_{n}}(1-r)^{b_{1}+\cdots+b_{n}-1} \int_{I^{n-1}} & \eta_{n-1}^{b_{n}-1} \prod_{i=1}^{n-2} \eta_{i}^{b_{i+1}} \\
& \times \prod_{i=1}^{n-1} y_{i}^{a_{i}-a_{i+1}-b_{i+1}} \prod_{i=1}^{n-1}\left(1-\xi_{i}\right)^{b_{i}-1} \bigwedge_{i=1}^{n-1} d \xi_{i} .
\end{aligned}
$$

Let us assume that the $a_{i}$ are arranged in decreasing order. Then

$$
\eta_{i}^{b_{i+1}} y_{i}^{a_{i}-a_{i+1}-b_{i+1}} \leq 1
$$

since $\eta_{i} \leq y_{i} \leq 1$. Therefore

$$
h(r) \leq r^{a_{n}}(1-r)^{b_{1}+\cdots+b_{n}-1} \int_{I^{n-1}} \eta_{n-1}^{b_{n}-1} y_{n-1}^{a_{n-1}-a_{n}-b_{n}} \prod_{i=1}^{n-1}\left(1-\xi_{i}\right)^{b_{i}-1} \bigwedge_{i=1}^{n-1} d \xi_{i} .
$$

Here four cases have to be discussed.

Case 1: $a_{n-1}-a_{n}-b_{n} \geq 0$. In this case

$$
\begin{aligned}
h(r) & \leq r^{a_{n}}(1-r)^{b_{1}+\cdots+b_{n}-1} \int_{I^{n-1}} \eta_{n-1}^{b_{n}-1} \prod_{i=1}^{n-1}\left(1-\xi_{i}\right)^{b_{i}-1} \bigwedge_{i=1}^{n-1} d \xi_{i} \\
& \leq r^{a_{n}}(1-r)^{b_{1}+\cdots+b_{n}-1} \prod_{i=1}^{n-1} \frac{\Gamma\left(b_{n}\right) \Gamma\left(b_{i}\right)}{\Gamma\left(b_{n}+b_{i}\right)} .
\end{aligned}
$$

Case 2: $a_{n-1}-a_{n}-b_{n}<0$ and $a_{n-1} \neq a_{n}$. Then

$$
\begin{aligned}
h(r) & \leq r^{a_{n}}(1-r)^{b_{1}+\cdots+b_{n}-1} \int_{I^{n-1}} y_{n-1}^{a_{n-1}-a_{n}-b_{n}} \eta_{n-1}^{b_{n}-1} \prod_{i=1}^{n-1}\left(1-\xi_{i}\right)^{b_{i}-1} \bigwedge_{i=1}^{n-1} d \xi_{i} \\
& \leq r^{a_{n}}(1-r)^{b_{1}+\cdots+b_{n}-1} \int_{I^{n-1}} \eta_{n-1}^{a_{n-1}-a_{n}-b_{n}} \eta_{n-1}^{b_{n}-1} \prod_{i=1}^{n-1}\left(1-\xi_{i}\right)^{b_{i}-1} \bigwedge_{i=1}^{n-1} d \xi_{i} \\
& \leq r^{a_{n}}(1-r)^{b_{1}+\cdots+b_{n}-1} \int_{I^{n-1}} \eta_{n-1}^{a_{n-1}-a_{n}-1} \prod_{i=1}^{n-1}\left(1-\xi_{i}\right)^{b_{i}-1} \bigwedge_{i=1}^{n-1} d \xi_{i} \\
& \leq r^{a_{n}}(1-r)^{b_{1}+\cdots+b_{n}-1} \prod_{i=1}^{n-1} \frac{\Gamma\left(a_{n-1}-a_{n}\right) \Gamma\left(b_{i}\right)}{\Gamma\left(a_{n-1}-a_{n}+b_{i}\right)} .
\end{aligned}
$$


Case 3: $a_{n-1}=a_{n}$. Choose an arbitrary $0<\epsilon \leq b_{n}$. Note that $y_{n-1} \geq r$ and $\eta_{n-1}>0$. Then

$$
\begin{aligned}
h(r) & \leq r^{a_{n}}(1-r)^{b_{1}+\cdots+b_{n}-1} \int_{I^{n-1}} y_{n-1}^{-b_{n}} \eta_{n-1}^{b_{n}-1} \prod_{i=1}^{n-1}\left(1-\xi_{i}\right)^{b_{i}-1} \bigwedge_{i=1}^{n-1} d \xi_{i} \\
& \leq r^{a_{n}}(1-r)^{b_{1}+\cdots+b_{n}-1} \int_{I^{n-1}} y_{n-1}^{-\epsilon} y_{n-1}^{\epsilon-b_{n}} \eta_{n-1}^{b_{n}-1} \prod_{i=1}^{n-1}\left(1-\xi_{i}\right)^{b_{i}-1} \bigwedge_{i=1}^{n-1} d \xi_{i} \\
& \leq r^{a_{n}}(1-r)^{b_{1}+\cdots+b_{n}-1} \int_{I^{n-1}} r^{-\epsilon} \eta_{n-1}^{\epsilon-b_{n}} \eta_{n-1}^{b_{n}-1} \prod_{i=1}^{n-1}\left(1-\xi_{i}\right)^{b_{i}-1} \bigwedge_{i=1}^{n-1} d \xi_{i} \\
& \leq r^{a_{n}}(1-r)^{b_{1}+\cdots+b_{n}-1} \int_{I^{n-1}} r^{-\epsilon} \eta_{n-1}^{\epsilon-1} \prod_{i=1}^{n-1}\left(1-\xi_{i}\right)^{b_{i}-1} \bigwedge_{i=1}^{n-1} d \xi_{i} \\
& =r^{a_{n}}(1-r)^{b_{1}+\cdots+b_{n}-1} r^{-\epsilon} \prod_{i=1}^{n-1} \frac{\Gamma(\epsilon) \Gamma\left(b_{i}\right)}{\Gamma\left(\epsilon+b_{i}\right)}
\end{aligned}
$$

Similarly to Section 2, this product of quotients of Gamma functions is meromorphic on the interval $\left[0, b_{n}\right]$ except at zero, where it has a pole of order $n-1$, and so there exists a constant $C$ such that

$$
\prod_{i=1}^{n-1} \frac{\Gamma(\epsilon) \Gamma\left(b_{i}\right)}{\Gamma\left(\epsilon+b_{i}\right)} \leq C \epsilon^{1-n} .
$$

Hence

$$
h(r) \ll r^{a_{n}}(1-r)^{b_{1}+\cdots+b_{n}-1} r^{-\epsilon} \epsilon^{1-n} .
$$

Now there are two subcases: If $r \leq e^{-1 / b_{n}}$, then $(\ln (1 / r))^{-1} \leq b_{n}$. In this case, we choose $\epsilon=(\ln (1 / r))^{-1}$ and obtain

$$
h(r) \ll r^{a_{n}}(1-r)^{b_{1}+\cdots+b_{n}-1} e(\ln (1 / r))^{n-1} .
$$

On the other hand, if $r \geq e^{-1 / b_{n}}$, we choose $\epsilon=b_{n}$ and obtain

$$
\begin{aligned}
h(r) & \ll r^{a_{n}}(1-r)^{b_{1}+\cdots+b_{n}-1} r^{-b_{n}} b_{n}^{1-n} \\
& \leq r^{a_{n}}(1-r)^{b_{1}+\cdots+b_{n}-1} e b_{n}^{1-n} .
\end{aligned}
$$

Combining (14) and (15) yields

$$
\begin{aligned}
h(r) & \ll r^{a_{n}}(1-r)^{b_{1}+\cdots+b_{n}-1}\left(e(\ln (1 / r))^{n-1}+e b_{n}^{1-n}\right) \\
& \ll r^{a_{n}}(1-r)^{b_{1}+\cdots+b_{n}-1}(\ln (1 / r))^{n-1} \quad \text { for all } 0<r<1 .
\end{aligned}
$$

This is enough for our purposes but we can get a more refined estimate as mentioned in the second case of Lemma A. Thus we reach the final case: 
Case 4: there exists $k$ such that $a_{k}>a_{k+1}=\cdots=a_{n}=a$. Let $F(r)$ be the Mellin convolution product of $f_{1}, f_{2}, \ldots, f_{k+1}$ and $G(r)$ be the Mellin convolution product of the rest, namely $f_{k+2} \ldots, f_{n}$. From the previous discussion it is clear that

$$
F(r) \ll r^{a}(1-r)^{b_{1}+\cdots+b_{k+1}-1}
$$

and

$$
G(r) \ll r^{a}(1-r)^{b_{k+2}+\cdots+b_{n}-1}(\ln (e / r))^{n-k-2} .
$$

Let $b=b_{1}+\cdots+b_{k+1}, d=b_{k+2}+\cdots+b_{n}$ and $n-k-1=l$. The case $l=1$ has been treated previously. So assume $l>1$. We see that

$$
\begin{aligned}
h(r) & =\left(F *_{M} G\right)(r) \\
& \ll \int_{r}^{1}(r / t)^{a}(1-r / t)^{b-1} t^{a}(1-t)^{d-1}\left(\ln \left(\frac{e}{t}\right)\right)^{l-1} \frac{d t}{t} \\
& \leq r^{a} \int_{r}^{1}(t-r)^{b-1} t^{-b}(1-t)^{d-1}\left(\ln \left(\frac{e}{t}\right)\right)^{l-1} \frac{d t}{t} .
\end{aligned}
$$

Now the change of variables $t=u+r-u r$ leads to $t-r=u(1-r), 1-t=$ $(1-u)(1-r), d t=(1-r) d u$ and

$$
\begin{aligned}
h(r) & \ll r^{a} \int_{0}^{1}(1-r)^{b-1} u^{b-1} t^{-b}(1-r)^{d-1}(1-u)^{d-1}\left(\ln \left(\frac{e}{t}\right)\right)^{l-1}(1-r) d u \\
& \leq r^{a}(1-r)^{b+d-1} \int_{0}^{1} u^{b-1} t^{-b}(1-u)^{d-1}\left(\ln \left(\frac{e}{t}\right)\right)^{l-1} d u .
\end{aligned}
$$

Noting that $t \geq u$ and $r>0$, and choosing an arbitrary $0<\epsilon \leq b$ implies

$$
\begin{aligned}
h(r) & \ll r^{a}(1-r)^{b+d-1} \int_{0}^{1} u^{b-1} t^{-\epsilon} t^{\epsilon-b}(1-u)^{d-1}\left(\ln \left(\frac{e}{t}\right)\right)^{l-1} d u \\
& \leq r^{a}(1-r)^{b+d-1} \int_{0}^{1} u^{b-1} r^{-\epsilon} u^{\epsilon-b}(1-u)^{d-1}\left(\ln \left(\frac{e}{t}\right)\right)^{l-1} d u \\
& \leq r^{a}(1-r)^{b+d-1} r^{-\epsilon} \int_{0}^{1} u^{b-1} u^{\epsilon-b}(1-u)^{d-1}\left(\ln \left(\frac{e}{u}\right)\right)^{l-1} d u \\
& \leq r^{a}(1-r)^{b+d-1} r^{-\epsilon} \int_{0}^{1} u^{\epsilon-1}(1-u)^{d-1}\left(\ln \left(\frac{e}{u}\right)\right)^{l-1} d u .
\end{aligned}
$$

Let $H_{j}(\epsilon)=\int_{0}^{1} u^{\epsilon-1}(1-u)^{d-1}(\ln u)^{j} d u$. This is the $j$-th derivative of the beta function $B(\epsilon, d)$ as a function of $\epsilon$, and $B(\epsilon, d)$ is holomorphic on $(-1, \infty)$ except at zero where it has a simple pole with residue 1 . This is easy to verify. So $\epsilon^{j+1} H_{j}(\epsilon)$ will be holomorphic on the interval $(-1, \infty)$. Observing that

$$
\int_{0}^{1} u^{\epsilon-1}(1-u)^{d-1}\left(\ln \left(\frac{e}{u}\right)\right)^{l-1} d u
$$


is a linear sum of the derivatives of order less than or equal to $l-1$ of the Beta function, we find

$$
\epsilon^{l} \int_{0}^{1} u^{\epsilon-1}(1-u)^{d-1}\left(\ln \left(\frac{e}{u}\right)\right)^{l-1} d u
$$

is bounded by a constant $C$ in the interval $[0, b]$. Thus

$$
h(r) \ll r^{a}(1-r)^{b+d-1} r^{-\epsilon} \epsilon^{-l} .
$$

Now arguing as in Case 3, if $r \leq e^{-1 / b}$, we choose $\epsilon=1 / \ln (1 / r)$ and get

$$
h(r) \ll r^{a}(1-r)^{b+d-1} e(\ln (1 / r))^{l}
$$

and if $r>e^{-1 / b}$, we let $\epsilon=b$, and have

$$
h(r) \ll r^{a}(1-r)^{b+d-1} e / b^{l} .
$$

Combining these two cases, we obtain

$$
h(r) \ll r^{a}(1-r)^{b+d-1}(\ln (e / r))^{l} .
$$

This fully proves Lemma A.

\section{Proof of Lemma B}

We recall (13):

$$
\begin{aligned}
h(r)=r^{a_{n}}(1-r)^{b_{1}+\cdots+b_{n}-1} \int_{I^{n-1}} \eta_{n-1}^{b_{n}-1} & \prod_{i=1}^{n-2} \eta_{i}^{b_{i+1}} \\
& \times \prod_{i=1}^{n-1} y_{i}^{a_{i}-a_{i+1}-b_{i+1}} \prod_{i=1}^{n-1}\left(1-\xi_{i}\right)^{b_{i}-1} \bigwedge_{i=1}^{n-1} d \xi_{i} .
\end{aligned}
$$

To make the differentiation easier, we introduce some notation. Let

$$
\begin{aligned}
& A=a_{n}, \quad B=b_{1}+\cdots+b_{n}, \\
& \eta=\left(\eta_{1}, \ldots, \eta_{n-1}\right), \quad \xi=\left(\xi_{1}, \ldots, \xi_{n-1}\right), \quad y=\left(y_{1}, \ldots, y_{n-1}\right), \\
& \alpha_{i}=a_{i}-a_{i+1}-b_{i+1} \quad \text { for } 1 \leq i \leq n-1 \text {, } \\
& \beta_{i}=b_{i+1} \quad \text { for } 1 \leq i \leq n-2, \quad \beta_{n-1}=b_{n}-1 \text {, } \\
& \beta=\left(\beta_{1}, \ldots, \beta_{n-1}\right), \quad G(\xi)=\prod_{i=1}^{n-1}\left(1-\xi_{i}\right)^{b_{i}-1}, \quad d \xi=\bigwedge_{i=1}^{n-1} d \xi_{i}, \quad J=I^{n-1} .
\end{aligned}
$$


With this notation and the multiindex notation as for example $y^{\alpha}=y_{1}^{\alpha_{1}} \cdots y_{n-1}^{\alpha_{n-1}}$, (13) can be written as

$$
h(r)=r^{A}(1-r)^{B-1} \int_{J} y^{\alpha} \eta^{\beta} G(\xi) d \xi .
$$

Clearly the function $\eta^{\beta} G\left(\xi_{i}\right)$ is summable $d \xi$, and each $y_{i}=\eta_{i}+r\left(1-\eta_{i}\right)$ satisfies $0<r \leq y_{i}<1$ for $0<r<1$. So one can differentiate under the integral sign with respect to $r$. But before we do that let us introduce the notation

$$
g_{1}(r)=r^{A}, \quad g_{2}(r)=(1-r)^{B-1}, \quad u_{i}=y_{i}^{\alpha_{i}} \quad \text { for } 1 \leq i \leq n-1 .
$$

Rewrite (16) as

$$
h(r)=\int_{J} g_{1} g_{2} u_{1} \cdots u_{n-1} \eta^{\beta} G(\xi) d \xi .
$$

Now differentiating under the integral sign, we obtain

$$
h^{(k)}(r)=\sum \int_{J} g_{1}^{\left(l_{1}\right)} g_{2}^{\left(l_{2}\right)} u_{1}^{\left(j_{1}\right)} \cdots u_{n-1}^{\left(j_{n-1}\right)} \eta^{\beta} G(\xi) d \xi,
$$

where the sum is over all $(n+1)$-tuples of nonnegative integers $\left(l_{1}, l_{2}, j_{1}, \ldots j_{n-1}\right)$ such that $k=l_{1}+l_{2}+j_{1}+\cdots+j_{n-1}$. Further it easy to check that

$$
\begin{aligned}
& u_{i}^{\left(j_{i}\right)}(r)=\alpha_{i}\left(\alpha_{i}-1\right) \cdots\left(\alpha_{i}-j_{i}+1\right) y_{i}^{\alpha_{i}-j_{i}}\left(1-\eta_{i}\right)^{j_{i}}, \\
& g_{1}^{\left(l_{1}\right)}(r)=A(A-1) \cdots\left(A-l_{1}+1\right) r^{A-l_{1}}, \\
& g_{2}^{\left(l_{2}\right)}(r)=(B-1)(B-2) \cdots\left(B-l_{2}\right)(-1)^{l_{2}}(1-r)^{B-l_{2}-1} .
\end{aligned}
$$

Since $y_{i} \geq r$ and $0 \leq \eta_{i} \leq 1$, the equalities above imply

$$
\begin{aligned}
& u_{i}^{\left(j_{i}\right)}(r) \ll y_{i}^{\alpha_{i}} r^{-j_{i}} \\
& g_{1}^{\left(l_{1}\right)}(r) \ll g_{1}(r) r^{-l_{1}} \\
& g_{2}^{\left(l_{2}\right)}(r) \ll(1-r)^{B-k-1}=g_{2}(r)(1-r)^{-k},
\end{aligned}
$$

where the last inequality is obtained because $0 \leq l_{2} \leq k$. From these three, we deduce that

$$
\begin{aligned}
g_{2}^{\left(l_{2}\right)}(r) g_{1}^{\left(l_{1}\right)}(r) u_{1}^{\left(j_{1}\right)}(r) \cdots u_{n-1}^{\left(j_{n-1}\right)}(r) \ll & g_{2}(r)(1-r)^{-k} g_{1}(r) u_{1}(r) \cdots \\
\cdots & u_{n-1}(r) r^{-l_{1}-j_{1}-\cdots-j_{n-1}} \\
\ll & r^{-k}(1-r)^{-k} g_{2}(r) g_{1}(r) u_{1}(r) \cdots u_{n-1}(r) .
\end{aligned}
$$

Multiplying both sides by $\eta^{\beta} G(\xi) d \xi$ and integrating over $J$ yield

$$
h^{(k)}(r) \ll r^{-k}(1-r)^{-k} h(r)
$$


and by Lemma A,

$$
h(r) \ll r^{A}(1-r)^{B-1}(\ln (e / r))^{n-1} .
$$

Hence we have

$$
h^{(k)}(r) \ll r^{A-k}(1-r)^{B-k-1}(\ln (e / r))^{n-1} .
$$

This proves Lemma B.

\section{Acknowledgment}

The authors would like to thank the referee for excellent suggestions.

\section{References}

[Čučković and Rao 1998] Z̆. Čučković and N. V. Rao, "Mellin transform, monomial symbols, and commuting Toeplitz operators", J. Funct. Anal. 154:1 (1998), 195-214. MR 99f:47033 Zbl 0936. 47015

[Louhichi 2007] I. Louhichi, "Powers and roots of Toeplitz operators", Proc. Amer. Math. Soc. 135:5 (2007), 1465-1475. MR 2007k:47043 Zbl 1112.47023

[Louhichi and Zakariasy 2005] I. Louhichi and L. Zakariasy, "On Toeplitz operators with quasihomogeneous symbols", Arch. Math. (Basel) 85:3 (2005), 248-257. MR 2006e:47061 Zbl 1088. 47019

[Remmert 1998] R. Remmert, Classical topics in complex function theory, Graduate Texts in Mathematics 172, Springer, New York, 1998. MR 98g:30002 Zbl 0895.30001

Received July 3, 2010. Revised December 18, 2010.

\section{ISSAM LOUHICHI}

DEPARTMENT OF MATHEMATICS AND STATistics

King FaHd University of PETROLEUM AND Minerals

DHAHRAN 31261

SAUDI ARABIA

issam@kfupm.edu.sa

N. V. RAO

DEPARTMENT OF MATHEMATICS

THE UNIVERSITY OF TOLEDO

MAIL STOP 942

TOLEDO, OHIO 43606-3390

UNITED STATES

rnagise@math.utoledo.edu 


\title{
PACIFIC JOURNAL OF MATHEMATICS
}

\author{
http://www.pjmath.org \\ Founded in 1951 by
}

E. F. Beckenbach (1906-1982) and F. Wolf (1904-1989)

\section{EDITORS}

V. S. Varadarajan (Managing Editor)

Department of Mathematics

University of California

Los Angeles, CA 90095-1555

pacific@math.ucla.edu

Vyjayanthi Chari

Department of Mathematics

University of California

Riverside, CA 92521-0135

chari@math.ucr.edu

\section{Robert Finn}

Department of Mathematics Stanford University

Stanford, CA 94305-2125

finn@math.stanford.edu

Kefeng Liu

Department of Mathematics

University of California

Los Angeles, CA 90095-1555

liu@math.ucla.edu
Darren Long

Department of Mathematics

University of California

Santa Barbara, CA 93106-3080

long@math.ucsb.edu

Jiang-Hua Lu

Department of Mathematics

The University of Hong Kong

Pokfulam Rd., Hong Kong jhlu@maths.hku.hk

Alexander Merkurjev

Department of Mathematics University of California

Los Angeles, CA 90095-1555 merkurev@math.ucla.edu
Sorin Popa

Department of Mathematics

University of California

Los Angeles, CA 90095-1555

popa@math.ucla.edu

Jie Qing

Department of Mathematics

University of California

Santa Cruz, CA 95064

qing@ cats.ucsc.edu

Jonathan Rogawski

Department of Mathematics

University of California

Los Angeles, CA 90095-1555

jonr@math.ucla.edu

\section{PRODUCTION}

pacific@math.berkeley.edu

Silvio Levy, Scientific Editor Matthew Cargo, Senior Production Editor

ACADEMIA SINICA, TAIPEI

CALIFORNIA INST. OF TECHNOLOGY

INST. DE MATEMÁTICA PURA E APLICADA

KEIO UNIVERSITY

MATH. SCIENCES RESEARCH INSTITUTE

NEW MEXICO STATE UNIV.

OREGON STATE UNIV.

\section{SUPPORTING INSTITUTIONS}

STANFORD UNIVERSITY
UNIV. OF BRITISH COLUMBIA
UNIV. OF CALIFORNIA, BERKELEY
UNIV. OF CALIFORNIA, DAVIS
UNIV. OF CALIFORNIA, LOS ANGELES
UNIV. OF CALIFORNIA, RIVERSIDE
UNIV. OF CALIFORNIA, SAN DIEGO
UNIV. OF CALIF., SANTA BARBARA

UNIV. OF CALIF., SANTA CRUZ

UNIV. OF MONTANA

UNIV. OF OREGON

UNIV. OF SOUTHERN CALIFORNIA

UNIV. OF UTAH

UNIV. OF WASHINGTON

WASHINGTON STATE UNIVERSITY

These supporting institutions contribute to the cost of publication of this Journal, but they are not owners or publishers and have no responsibility for its contents or policies.

See inside back cover or www.pjmath.org for submission instructions.

The subscription price for 2011 is US \$420/year for the electronic version, and \$485/year for print and electronic.

Subscriptions, requests for back issues from the last three years and changes of subscribers address should be sent to Pacific Journal of Mathematics, P.O. Box 4163, Berkeley, CA 94704-0163, U.S.A. Prior back issues are obtainable from Periodicals Service Company, 11 Main Street, Germantown, NY 12526-5635. The Pacific Journal of Mathematics is indexed by Mathematical Reviews, Zentralblatt MATH, PASCAL CNRS Index, Referativnyi Zhurnal, Current Mathematical Publications and the Science Citation Index.

The Pacific Journal of Mathematics (ISSN 0030-8730) at the University of California, c/o Department of Mathematics, 969 Evans Hall, Berkeley, CA 94720-3840, is published monthly except July and August. Periodical rate postage paid at Berkeley, CA 94704, and additional mailing offices. POSTMASTER: send address changes to Pacific Journal of Mathematics, P.O. Box 4163, Berkeley, CA 94704-0163.

PJM peer review and production are managed by EditFLOW ${ }^{\mathrm{TM}}$ from Mathematical Sciences Publishers.

PUBLISHED BY PACIFIC JOURNAL OF MATHEMATICS

at the University of California, Berkeley 94720-3840

A NON-PROFIT CORPORATION

Typeset in IATEX

Copyright $(2011$ by Pacific Journal of Mathematics 


\section{PACIFIC JOURNAL OF MATHEMATICS}

Volume $252 \quad$ No. $1 \quad$ July 2011

Some dynamic Wirtinger-type inequalities and their applications

RaVi P. Agarwal, Martin Bohner, Donal O'REgAn and SAMIR H.

SAKER

Splitting criteria for vector bundles on higher-dimensional varieties

PARSA BAKHTARY

Average Mahler's measure and $L_{p}$ norms of unimodular polynomials

KWOK-KWOng STEPHen CHOI and Michael J. MossinghofF

Tate resolutions and Weyman complexes

David A. CoX and EVgeny Materov

On pointed Hopf algebras over dihedral groups

FERNANDO FANTINO and GASTON ANDRÉS GARCIA

Integral topological quantum field theory for a one-holed torus

PATRicK M. Gilmer and Gregor Masbaum

Knot 4-genus and the rank of classes in $\boldsymbol{W}(\mathbb{Q}(t))$

CHARLES LIVINGSTON

Roots of Toeplitz operators on the Bergman space

ISSAM LOUHICHI and NAGISETTY V. RAO

Uniqueness of the foliation of constant mean curvature spheres in asymptotically 145 flat 3-manifolds

SHIGUANG MA

On the multiplicity of non-iterated periodic billiard trajectories

MARCo MAZZUCCHELLI

A remark on Einstein warped products

MiCHELE RIMOLDI

Exceptional Dehn surgery on large arborescent knots

YING-QING WU

Harnack estimates for the linear heat equation under the Ricci flow 